\title{
Sarcoidose testicular. Um diagnóstico a ser considerado
}

\section{Testicular sarcoidosis. A diagnosis to be considered}

\author{
Vanderlei Real ${ }^{1}$; Guilherme Locks de Loyola ${ }^{2}$; Paulo Eduardo Zanoni ${ }^{2}$; Lara Flório Real ${ }^{3}$
}

\section{INTRODUÇÃO}

\begin{abstract}
A sarcoidose é uma doença multissistêmica, idiopática, caracterizada pela presença de granulomas nãocaseosos. Embora possa ter uma apresentação variada, na sua forma clássica a doença compromete o pulmão e os linfonodos hilares, o que ocorre em cerca de $85-90 \%$ dos $\operatorname{casos}^{1,2}$. O acometimento do sistema urogenital é extremamente raro ${ }^{1,2}$. O curso clínico da sarcoidose pode apresentar-se sob diversos aspectos, podendo variar de doença assintomática com resolução espontânea até doença progressiva com insuficiência de órgãos ou mesmo morte.
\end{abstract}

\section{RELATO DO CASO}

Um homem de 55 anos, branco, procurou o nosso serviço com queixa de desconforto testicular bilateral há três meses. Referia ainda perda de peso e ansiedade. O paciente não fazia uso de nenhum medicamento e tinha como único dado relevante na sua história médica, um episódio de sarcoidose pulmonar há 11 anos, que foi tratada com corticoesteróides e teve cura comprovada. O exame físico não revelou anormalidades, os testículos apresentavam superfície e tamanhos normais, não havia linfonodos palpáveis, a PA estava normal e o exame digital da próstata e reto não apresentou alterações. A ultrassonografia testicular evidenciava testículos com dimensões normais, contornos regulares, identificando nódulos hipoecóicos, medindo de 3 a $8 \mathrm{~mm}$ em ambos testículos (Figura 1). DHL, alfa-feto proteína e beta-HCG estavam dentro dos limites da normalidade. As tomografias computadorizadas de tórax, abdome e pelve também não apresentavam alterações.

Optamos pela abordagem cirúrgica via inguinal com clampeamento alto do cordão espermático. Como a superfície testicular era lisa, realizamos a orquiectomia diagnóstica. No exame macroscópico não havia alterações. Aos cortes viam-se dois nódulos branco-acinzentados medindo $0,4 \mathrm{~cm}$ o de maior diâmetro. O exame histopatológico concluiu que existia inflamação crônica granulomatosa nãocaseificante focal (Figura 2 - A e B). A pesquisa para fun- gos e BAAR foi negativa, confirmando diagnóstico de granuloma sarcoide. Frente ao diagnóstico de sarcoidose testicular e da exclusão de processos malignos, optamos pelo acompanhamento clínico do paciente.

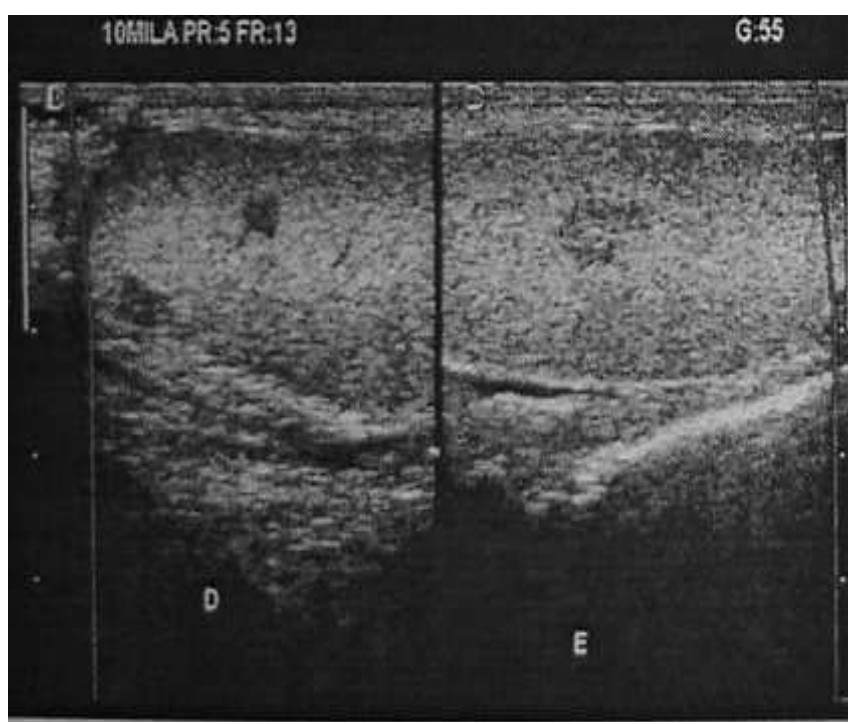

Figura 1 - Ultrassonografia evidenciando nódulos hipoecóicos de contornos irregulares em ambos testículos.
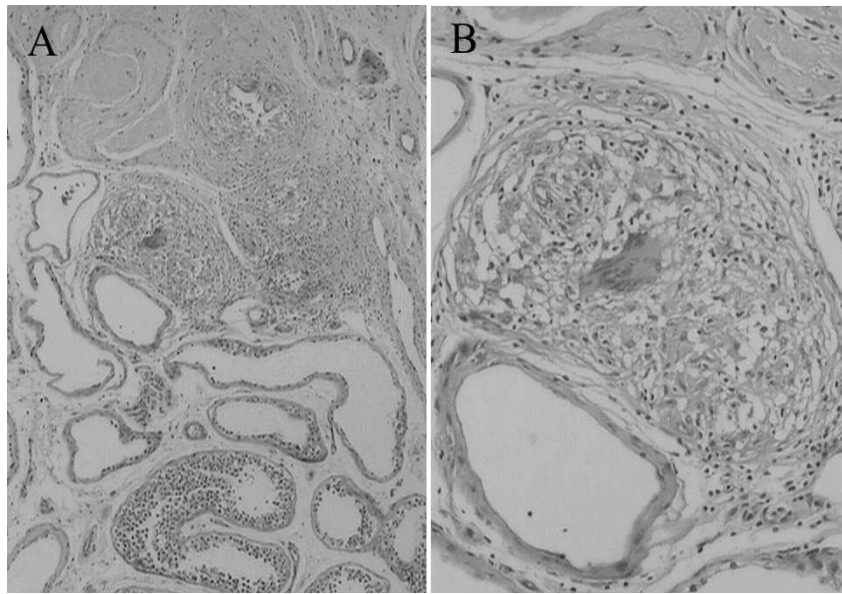

Figura 2

A) Corte histológico de testículo evidenciando inflamação crônica granulomatosa não-caseificante focal (HE, X40). B) Magnificação da área do granuloma $(H E, X 100)$.

Trabalho realizado no Hospital Beneficência Portuguesa de Pelotas - RS-BR.

1. Urologista do Hospital Beneficência Portuguesa de Pelotas - RS-BR; 2. R4 de Urologia do Hospital Governador Celso Ramos - Florianópolis - SCBR; 3. R4 de Cirurgia Torácica da Santa Casa de Misericórdia de Porto Alegre - RS-BR; 4. R2 de Gineco-Obstetricia do Hospital Nossa Senhora de Conceição de Porto Alegre - RS-BR. 
Após um ano de seguimento o paciente se apresentava assintomático e sem sintomas de progressão da doença.

\section{DISCUSSÃO}

A sarcoidose é uma doença multissistêmica, mas em cerca de $85 \%$ dos casos o comprometimento está localizado no tórax, enquanto o comprometimento urogenital ocorre em apenas $0,2 \%$ dos casos diagnosticados clinicamente e em até $5 \%$ daqueles diagnosticados por autópsia1. Segundo as bases de dados pesquisadas, incluindo o presente caso, existem 62 casos de acometimento urogenital pela sarcoidose comprovados histologicamente, sendo que 29 apresentavam comprometimento testicular ${ }^{1,2}$.

Duas condutas principais são citadas quando se trata do acometimento geniturinário, ainda não existindo um consenso. Singer et al. sugerem que a melhor abordagem terapêutica é a orquiectomia radical, baseado na gran- de associação entre câncer testicular e sarcoidose, além da presença de reação granulomatosa em muitos casos de seminoma, teratoma, teratocarcinoma e carcinoma embrionário ${ }^{3}$. Em contrapartida, outros autores acreditam que a orquiectomia seja uma terapia inicial muito agressiva, devido a grande quantidade de condições benignas associadas. Eles sugerem a biopsia guiada por ultrassom para estabelecer o diagnóstico, reservando a orquiectomia para casos de malignidade e quando o diagnóstico for indeterminado ${ }^{1,2}$.

Apesar de ser incomum, a sarcoidose urogenital tem que ser sempre considerada como um diagnóstico diferencial em caso de massa testicular. Um diagnóstico préoperatório com alguma certeza ocasionalmente é possível, mas na maioria dos casos não. Devemos sempre considerar processos malignos, mesmo em caso de sarcoidose sistêmica e recomendamos orquiectomia no caso de um paciente com massa intratesticular unilateral. Frente ao diagnóstico de condição benigna, a conduta mais conservadora possível deve ser estabelecida.

\section{A B S T R A C T}

Sarcoidosis is a disease of unknown etiology, characterized by the presence of noncaseating granulomas in multiple organs. We present a case of testicular sarcoidosis in a white, 55-year-old man who has come to our department complaining of bilateral testicular discomfort and weight loss.

Key words: Sarcoidosis. Testicular diseases. Granuloma. Male.

\section{REFERENCIAS}

1. Kodama K, Hasegawa T, Egawa M, Tomosugi N, Mukai A, Namiki M. Bilateral epididymal sarcoidosis presenting without radiographic evidence of intrathoracic lesion: Review of sarcoidosis involving the male reproductive tract. Int J Urol 2004; 11(5):345-8.

2. Massarweh NN, Bhalani VK, Shaw KK, Crawford B, Lang E, Davis R. Testicular presentation of sarcoidosis and organ preservation: case report and review of management strategies. Urology 2006; 67(1):200.

3. Singer AJ, Gavrell GJ, Leidich RB, Quinn AD. Genitourinary involvement of systemic sarcoidosis confined to testicle. Urology 1990; 35(5):442-4.
Recebido em 13/02/2007

Aceito para publicação em 15/03/2007

Conflito de interesse: nenhum

Fonte de financiamento: nenhuma

\section{Como citar este artigo:}

Real V, Loyola GL, Zanoni PE, Real LF. Sarcoidose testicular. Um diagnóstico a ser considerado. Rev Col Bras Cir. [periódico na Internet] 2011; 38(2). Disponível em URL: http://www.scielo.br/rcbc

Endereço para correspondência:

Vanderlei Rosas Real

E-mail: vanderlei.real@gmail.com 